\title{
Left ventricular assist devices for less sick patients: A roadmap for the future or a fork in the road?
}

\author{
Sumeet S. Mitter, MD, MSc, and Sean P. Pinney, MD
}

\footnotetext{
From the Zena and Michael A. Wiener Cardiovascular Institute, Icahn School of Medicine at Mount Sinai, New York, NY.

Received for publication Aug 9, 2018; revisions received Nov 28, 2018; accepted for publication Dec 10, 2018; available ahead of print March 14, 2019.

Address for reprints: Sean P. Pinney, MD, Zena and Michael A. Wiener Cardiovascular Institute, Icahn School of Medicine at Mount Sinai, 1190 5th Ave, Box 1030, New York, NY 10029 (E-mail: sean.pinney@mssm.edu). J Thorac Cardiovasc Surg 2019;158:167-9

$0022-5223 / \$ 36.00$

Copyright (C) 2019 by The American Association for Thoracic Surgery

https://doi.org/10.1016/j.jtcvs.2018.12.106
}

Feature Editor's Introduction-Durable left ventricular assist device (LVAD) therapy has long been touted as the solution to the growing number of patients with advanced heart failure. Despite widespread adoption of LVAD therapy, the therapy has remained largely limited to patients with advanced heart failure requiring intravenous inotropes. Inroads to less ill populations of patients with heart failure, long the holy grail of LVAD therapy, has been slow to gain traction. ${ }^{1}$ Mitter and Pinney provide an insightful commentary on the state of durable LVAD therapy for treatment of heart failure in patients with less-advanced symptoms than traditionally referred for device therapy. Mitter and Pinney make a controversial statement: "Yet at this point in time, the technology has not lived up to its full potential." This statement made by the authors is likely to conjure a great debate on the issue. Some might argue that this therapy has offered meaningful improvement in quality of life and longevity to thousands of patients. Others would argue that this therapy, in reality, has been associated with a significant adverse event burden and has not lived up to the anticipated hype promised nearly more than a decade ago with the first approval of continuous-flow technology for bridge to transplant indication in 2008 and for destination therapy in 2010 in the United States. Whichever side of the debate one might fall, the commentary by Mitter and Pinney provides a cogent portrayal of state of LVAD therapy for the less ill and the complex decisions both providers and patients need to make when assessing the benefits and risks of this therapy.

When you come to a fork in the road, take it. -Yogi Berra ${ }^{2}$

It has been 10 years since the first implantable rotary blood pump was approved for commercial use. ${ }^{3}$ In that time, the field of mechanical circulatory support has experienced a transformation manifested by an improvement in clinical

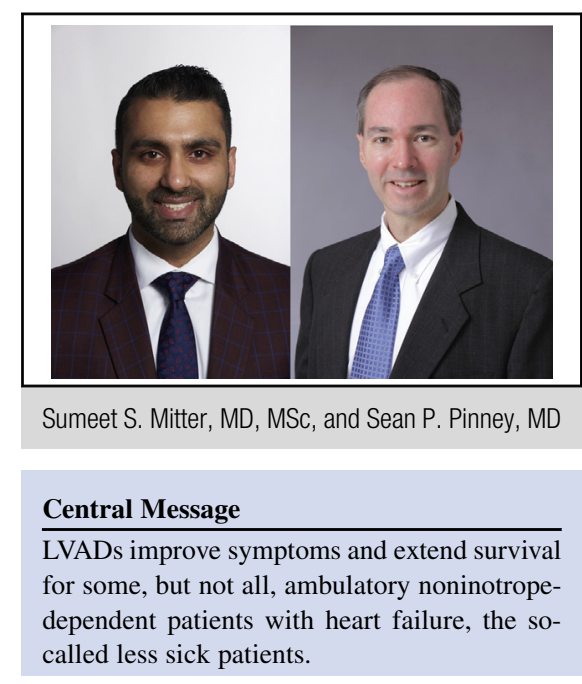

See Commentary on page 170 .

outcomes, an increase in implant volumes, and the realization of meaningful patient survival. The use of left ventricular assist devices (LVADs) for lifelong support, so-called destination therapy, has become the treatment of choice for nontransplant eligible patients with advanced heart failure. ${ }^{4}$ These patients, who before 2010 had no access to a durable pump, are the fastest growing population of LVAD recipients with annual implant volumes exceeding that of heart transplantation. ${ }^{5}$ Further refinements in pump technology, including the use of full mag-lev technology, have reduced the adverse event burden that patients experience while still delivering meaningful improvements in functional capacity and quality of life. ${ }^{6}$

Yet at this point in time, the technology has not lived up to its full potential. The early experiences with continuousflow LVADs, which could rescue patients in cardiogenic shock and restore them to a viable life, naturally led surgeons and cardiologists to consider expanding their use in other populations including the elderly and in less sick populations. ${ }^{7}$ Although there has been unquestioned anecdotal success in selected elderly populations, it is the latter group that has generated the most controversy. ${ }^{8}$ These ambulatory noninotrope-dependent patients with advanced heart failure, typically classified as being Interagency Registry for Mechanically Assisted Circulatory Support (INTERMACS) profiles 4 to 7 , have an unacceptably high 1-year mortality $(40 \%, 26 \%$, and $16 \%$ for INTERMACS profile 4, 5, and 6-7, respectively) and significant symptom burden (including limited mobility, significant pain/ 
discomfort, and an inability to perform self-care according to the EuroQol health-related quality of life instrument). ${ }^{9}$ Yet the use of LVADs in this patient population has not grown as rapidly as originally expected in part because of the unacceptably high adverse event rate and recurring hospitalizations associated with this technology. We are learning that some patients, particularly those with a tolerable level of exercise impairment, choose to delay surgery and avoid the burdens of living on support despite being told that their survival is likely to be improved by selecting LVAD surgery. ${ }^{10}$ Providers also may shy away from recommending such an invasive therapy so as not to sound alarmist to patients with less severe symptoms and to delay shared-decision conversations that need a proper amount of time and multidisciplinary participation to be successful. In others, there is an anticipated need for biventricular support to achieve a desired improvement in functional capacity and quality of life that may not be achievable with univentricular support alone.

Recently published clinical trials have helped to illuminate this complex dilemma of electively choosing LVADs in less sick patients. ${ }^{1,11,12}$ The original Risk Assessment and Comparative Effectiveness of Left Ventricular Assist Device and Medical Management in Ambulatory Heart Failure Patients (ROADMAP) was a nonrandomized, observational trial that evaluated noninotrope-dependent ambulatory patients with heart failure who were eligible to receive a continuous-flow LVAD, in this case the HeartMate II (Abbott Laboratories, Abbott Park, Ill). ${ }^{11}$ Entry criteria included at least 1 hospitalization or 2 emergency department visits in the past 12 months and a 6-minute walk distance (6MWD) of less than $300 \mathrm{~m}$ and otherwise met eligibility criteria for a destination therapy LVAD (ejection fraction $<25 \%$ and receiving optimal medical therapy). Patients decided at the time of enrollment whether to move forward with LVAD implantation or to continue with optimal medical management (OMM). The primary composite outcome was survival on original therapy at 1 year with improvement in 6MWD greater than $75 \mathrm{~m}$. At 1 year, intention-to-treat survival was similar in the 2 groups largely because of delayed LVAD implantation in the OMM group. As-treated event-free survival was significantly higher for the LVAD group who also achieved greater functional capacity and quality of life scores.

Although the trial design led to an imbalance in patient characteristics between the 2 study groups, it did produce meaningful insights into patient decision-making. In general, those choosing to receive an LVAD were more symptomatic with a lower 6MWD and more likely to be INTERMACS profile 4. Those choosing OMM had better perceived health-related quality of life and were more likely to be INTERMACS profile 5 to 7 . Of note, those choosing to delay LVAD therapy did not appear to pay a price in terms of survival, but also did not experience the improvements in functional capacity and quality of life enjoyed by those who selected LVAD support at an earlier time point.

A recent publication from the ROADMAP investigators probed a bit deeper into the outcomes for patients with INTERMACS profile 4 versus those with profiles 5 to $7 .^{1}$ Shah and colleagues ${ }^{1}$ highlight that more INTERMACS profile 4 patients met the primary composite end point (1-year survival on original therapy with improved 6MWD) with LVAD implantation compared with OMM, but such was not the case for those patients with INTERMACS profile 5 to 7. Not only was there no statistically significant difference in survival for those receiving an LVAD but also there was also no apparent improvement in functional capacity, quality of life, or depression in these less sick individuals.

Shah and colleagues ${ }^{1}$ point out that there is a clear divide in the impact durable mechanical support can offer less sick patients, and this separation arises between INTERMACS profiles 4 and 5 . Why might that be? One reason may be because patients who are truly profile 4, namely, symptomatic at rest, appear much different than those who are otherwise ambulatory with varying degrees of impairment (profiles 5-7). These profile 4 patients seem to align more with those who are profile 3 . In one center they may be placed on an inotrope and in another perhaps a vasodilator or simply asked to hold on and continue with medical therapy. That leaves a fair amount of overlap for profiles 5 to 7 . As Cowger and colleagues ${ }^{13}$ showed, there is great heterogeneity in how surgeons, cardiologists, and coordinators view disease severity in their patients, but most can differentiate inotrope from no-inotrope and ambulatory symptoms versus rest symptoms.

One additional question raised by Shah and colleagues' article $^{1}$ is that as a medical community we need to ask what costs are tolerable in the attempt to improve the lives of less sick patients with heart failure. Durable mechanical circulatory support implantation cannot be divorced from its adverse event profile. In the current study, regardless of INTERMACS profile, gastrointestinal bleeding remained a vexing problem and led to hospital readmissions. ${ }^{1}$ This in turn lowered patients' quality of life, deepened depression, and placed a financial burden on the health care system. A recent cost-effectiveness analysis by Baras Shreibati and colleagues ${ }^{14}$ estimated the incremental cost-effectiveness ratio (ICER) of LVAD implantation in ambulatory patients with heart failure to be $\$ 209,400$ per each quality-adjusted life-year gained among Medicare beneficiaries. ${ }^{14}$ Notably, in today's dollars, an ICER of $\$ 100,000$ to $\$ 150,000$ is the accepted norm for incremental benefit. Implantation of destination therapy LVAD in ambulatory patients with heart failure should be considered a less cost-effective strategy for patient and disease management. On the other hand, a 50\% drop in readmission rates coupled with a reduction of postimplantation costs by $\$ 51,000$ would lower the ICER to $\$ 86,900$, thus making destination therapy LVAD therapy 
cost-effective in ambulatory heart failure. As part of the ongoing MOMENTUM 3 trial, comparing the centrifugal flow HeartMate 3 (Abbott Laboratories) and the HeartMate II LVAD, a prespecified cost-effectiveness analysis was written into the study protocol. ${ }^{15}$ This analysis showed that the HeartMate 3 device-associated event-driven rehospitalizations were significantly less expensive than HeartMate II rehospitalizations, yielding a reduction in cost by approximately $50 \%$ per patient-year. In real-world practice outside that of a trial setting, it is feasible then that with newer LVAD technology, implantation in ambulatory patients with heart failure will eventually prove to be cost-effective.

After reading Shah and colleagues' article, ${ }^{1}$ we are left with a remaining question stemming from the ROADMAP experience: "So, who is in the driver's seat and what will it take to increase LVAD use in less sick patients?" The short answer is that less symptomatic patients, more than providers, are the key decision makers. What they seek is a device that improves symptoms as much as it extends survival, one that has a low adverse event rate, and one that enhances rather than interferes with quality of life. Cast in this light, it seems that LVAD use in less sick patients still faces a long road ahead. Use of the HeartMate 3 LVAD may have been more cost-effective than its predecessors in a clinical trial population, but it did not reduce 2-year bleeding events or driveline-associated infections. ${ }^{16}$ Targeting a lower therapeutic international normalized ratio, as is being done in the MAGENTUM trial, has the potential to safely lower bleeding rates. ${ }^{17}$ It is a matter of speculation whether the outcomes with ROADMAP would have been different if the HeartMate 3 was the pump being implanted rather than the HeartMate II, which is associated with higher rates of stroke and pump thrombosis. This lack of hemocompatibility coupled with the obligate need for an external driveline continues to limit the wider dissemination of this therapy to a broader swath of patients with heart failure. The technology to support fully implantable left ventricular assist systems currently exists, but device reliability and tradeoffs between battery size and untethered time have slowed its development. ${ }^{18}$ Achieving fully implantable left ventricular assist systems could make device implantation more appealing to ambulatory INTERMACS class 4 patients and select INTERMACS class 5 to 7 patients. Whether it will remains a matter of speculation.

Just as all roads lead to Rome, so too is it that all patients with heart failure, barring some other calamity along the way, will ultimately progress to end-stage failure. We as providers can only inform those ambulatory, noninotropedependent patients that when their symptoms are advanced and quality of life is poor they must eventually come to the fork in the road and decide whether to accept an LVAD. Which path they choose is up to them.

\section{Conflict of Interest Statement}

S.P.P. reports consulting fees and honoraria from Abbott Inc, CareDx, and Medtronic Inc, and is on the Advisory Board of Alnylam. S.S.M has nothing to disclose with regard to commercial support.

\section{References}

1. Shah KB, Starling RC, Rogers JG, Horstmanshof DA, Long JW, Kasirajan V, et al. Left ventricular assist devices versus medical management in ambulatory heart failure patients: an analysis of INTERMACS Profiles 4 and 5 to 7 from the ROADMAP study. J Heart Lung Transplant. 2018;37:706-14.

2. Berra Y, Kaplan D. When You Come to a Fork in the Road, Take It!: Inspiration and Wisdom from One of Baseball's Greatest Heroes. New York: Hachette Books; 2001.

3. Miller LW, Pagani FD, Russell SD, John R, Boyle AJ, Aaronson KA, et al. Use of a continuous-flow device in patients awaiting heart transplantation. $N$ Engl $J$ Med. 2007;357:885-96.

4. Pinney SP, Anyanwu AC, Lala A, Teuteberg JJ, Uriel N, Mehra MR. Left ventricular assist devices for lifelong support. J Am Coll Cardiol. 2017;69:2845-61.

5. Kirklin JK, Pagani FD, Kormos RL, Stevenson LW, Blume ED, Myers SL, et al. Eighth annual INTERMACS report: special focus on framing the impact of adverse events. J Heart Lung Transplant. 2017;36:1080-6.

6. Mehra MR, Naka Y, Uriel N, Goldstein DJ, Cleveland JC Jr, Colombo PC, et al. A fully magnetically levitated circulatory pump for advanced heart failure. $N$ Engl J Med. 2017:376:440-50.

7. Pagani FD, Aaronson KD, Kormos R, Mann DL, Spino C, Jeffries N, et al. The NHLBI REVIVE-IT study: understanding its discontinuation in the context of current left ventricular assist device therapy. J Heart Lung Transplant. 2016; 35:1277-83.

8. Adamson RM, Stahovich M, Chillcott S, Baradarian S, Chammas J, Jaski B, et al. Clinical strategies and outcomes in advanced heart failure patients older than 70 years of age receiving the HeartMate II left ventricular assist device: a community hospital experience. J Am Coll Cardiol. 2011;57:2487-95.

9. Stewart GC, Kittleson MM, Patel PC, Cowger JA, Patel CB, Mountis MM, et al. INTERMACS (interagency registry for mechanically assisted circulatory support) profiling identifies ambulatory patients at high risk on medical therapy after hospitalizations for heart failure. Circ Heart Fail. 2016;9:e003032.

10. Allen LA, McIlvennan CK, Thompson JS, Dunlay SM, LaRue SJ, Lewis EF, et al. Effectiveness of an intervention supporting shared decision making for destination therapy left ventricular assist device: the DECIDE-LVAD randomized clinical trial. JAMA Intern Med. 2018;178:520-9.

11. Estep JD, Starling RC, Horstmanshof DA, Milano CA, Selzman CH, Shah KB, et al. Risk assessment and comparative effectiveness of left ventricular assist device and medical management in ambulatory heart failure patients. $J$ Am Coll Cardiol. 2015;66:1747-61.

12. Starling RC, Estep JD, Horstmanshof DA, Milano CA, Stehlik J, Shah KB, et al. Risk assessment and comparative effectiveness of left ventricular assist device and medical management in ambulatory heart failure patients: the ROADMAP study 2-year results. JACC Heart Fail. 2017;5:518-27.

13. Cowger J, Shah P, Stulak J, Maltais S, Aaronson KD, Kirklin JK, et al INTERMACS profiles and modifiers: heterogeneity of patient classification and the impact of modifiers on predicting patient outcome. J Heart Lung Transplant. 2016;35:440-8.

14. Baras Shreibati J, Goldhaber-Fiebert JD, Banerjee D, Owens DK, Hlatky MA Cost-effectiveness of left ventricular assist devices in ambulatory patients with advanced heart failure. JACC Heart Fail. 2017;5:110-9.

15. Mehra MR, Salerno C, Cleveland JC, Pinney S, Yuzefpolskaya M, Milano CA, et al. Health care resource use and cost implications in the MOMENTUM 3 long-term outcome study: a randomized controlled trial of a magnetically levitated cardiac pump in advanced heart failure. Circulation. 2018;138:1923-34.

16. Mehra MR, Goldstein DJ, Uriel N, Cleveland JC Jr, Yuzefpolskaya M, Salerno C, et al. Two-year outcomes with a magnetically levitated cardiac pump in heart failure. N Engl J Med. 2018;378:1386-95.

17. Netuka I, Ivák P, Tucanová Z, Gregor S, Szárszoi O, Sood P, et al. Evaluation of low-intensity anti-coagulation with a fully magnetically levitated centrifugalflow circulatory pump-the MAGENTUM 1 study. J Heart Lung Transplant. 2018;579-86.

18. Mallidi HR, Anand J, Cohn WE. State of the art of mechanical circulatory support. Tex Heart Inst J. 2014;41:115-20. 